\title{
Dynamic behaviors of a discrete-time predator-prey bioeconomic system
}

\author{
Weiyi Liu*, Donghan Cai and Jie Shi
}

\section{"Correspondence:}

weiyiliu@whu.edu.cn

School of Mathematics and

Statistics, Wuhan University, Wuhan,

P.R. China

\section{Springer}

\begin{abstract}
Bifurcation and chaotic behavior of a discrete-time singular bioeconomic system are investigated. First, the traditional catch equation is modified after accounting for the handling time of the catch in a singular bioeconomic system. To discover the richer dynamics compared with the continuous form, the proposed system is considered difference scheme. Specially, the tangent space local parameterization condensed method for DAEs is generalized. The new local parameterization method is sufficiently general to be applicable to this type of discrete singular system. Also the dynamic behaviors of the system are investigated, by using normal form theory, center manifold theorem and bifurcation theory, it is shown that the system undergoes a Neimark-Sacker bifurcation and a flip bifurcation, on varying step-size in some range. In addition, numerical simulations are presented not only to illustrate our results with the theoretical analysis, but also to exhibit the complex dynamical behaviors.
\end{abstract}

MSC: Primary 37G10; secondary 39A28; 39A30

Keywords: Generalized local parameterization method; Discrete singular system; Bioeconomic system; Nonlinear harvesting rate; Stability; Codimension-one bifurcation; Chaotic behavior

\section{Introduction}

The study of bioeconomics has a very long history. Bioeconomics is closely related to the early development of theories in fishery economics. The basic model due to Gordon (1954) and Schaefer (1957) is

$$
\frac{d x}{d t}=G(x)-h(x, E)
$$

and the instantaneous profit rate is

$$
v=p h(x, E)-c E,
$$

where the "catch equation"

$$
h(x, E)=q E x
$$

is called the Schaefer catch-effort relation [1]. $x$ is the biomass at time $t . E$ is the harvesting effort, in the fishery setting, which is typically specified as the number of (standard) vessels

(c) The Author(s) 2018. This article is distributed under the terms of the Creative Commons Attribution 4.0 International License (http://creativecommons.org/licenses/by/4.0/), which permits unrestricted use, distribution, and reproduction in any medium, provided you give appropriate credit to the original author(s) and the source, provide a link to the Creative Commons license, and indicate if changes were made. 
actively fishing at time $t$. The units of harvesting effort would then be Standard Vessel Units (SVU). $c$ is the cost of effort, and $p$ is the ex-vessel price of fish. The parameter $q$ is a constant, called catchability, units of $q$ are $\mathrm{SVU}^{-1} \times(\text { time unit })^{-1}$. Thus, $q$ represents the proportion of the current stock $x$ caught by one standard vessel in one time unit (to normalize units, one could let $q=1$ ). To be specific we will now use time units of days (rather than years). Thus $G(x)$ now represents the population growth per day and $h$ is the daily harvesting rate, both being measured in tonnes per day.

Predator-prey models with harvesting is always a hot research topic (see [2-9]). The most general form of the two-dimensional predator-prey model is Kolmogorov's model [10]:

$$
\left\{\begin{array}{l}
\dot{x}=x f(x, y), \\
\dot{y}=y g(x, y),
\end{array}\right.
$$

here $x$ and $y$ denote the prey and predator populations at time $t$, respectively. In recent years, to analyze this model from an economic point of view, many researchers have studied a class of bioeconomic systems which combine system (1.3) with prey (or predator) harvesting and (1.1), and obtained some excellent results, such as the stability of equilibria, limit cycle, Hopf bifurcation, saddle-node bifurcation, singular induced bifurcation (see $[4-6,8,9,11,12])$. It is given by

$$
\left\{\begin{array}{l}
\dot{x}=x f(x, y)-h(x, E), \\
\dot{y}=y g(x, y), \\
v=p h(x, E)-c E,
\end{array}\right.
$$

here $E$ is the effort applied to harvesting the prey at time $t$. The extended model is a system of differential-algebraic equations (DAEs) with differential variables $x, y$ and algebraic variable $E$. In these literatures, $h(x, E)$ is traditionally taken as $q E x$. However, the Schaefer catch-effort relation has some flaws as Ref. [13] points out:

(i) All processes affecting stock productivity (e.g., growth, mortality and recruitment) are subsumed in the effective relationship between effort and catch. This hypothesis has been proved to be not reasonable.

(ii) The catchability coefficient $q$ is not always constant. Improvements in technology and fishing power make $q$ often vary through time.

(iii) The harvesting function did not account for the handling time of the catch and the competition between standard vessels which are utilized for harvesting of resource. On the contrary, evidence for a nonlinear harvest function is quite strong for a number of stocks [14].

In economics there is a tradition of formulating the dynamical systems as processes in discrete time from the outset, as difference equations instead of as differential equations. The cobweb model, the Cournot duopoly model, and the Samuelson-Hicks business cycle model are all examples of this tradition. On the other hand, in population ecology many authors are more focused on discrete-time models for the following reasons: one is that, for nonoverlapping generations, births occur in regular, well-defined "breeding seasons". This contradicts the assumption that births occur continuously [15]. The other is that the 
discrete-time models can also govern efficient computing models for continuous ones in order to achieve the numerical emulation. However, as far as we know, there are few studies of the difference scheme of system (1.4) which represents a class of discrete singular systems and no one has considered the general method to be applied to deal with it by now.

The main objective of this paper is to investigate the dynamic behaviors of a discretetime economic predator-prey model. The model, featuring a more realistic nonlinear harvesting function, will give rise to some interesting dynamic behaviors, as we see in Sect. 4 . The paper is organized as follows. We develop a local parameterization method based on the tangent space local parameterization of DAEs in [16] and use it to deal with the aforementioned discrete singular system in the next section. In Sect. 3, we analyze the stability of the fixed point. In addition, we also classify the fixed point based on the geometric properties of trajectories distribution near it. Surprisingly, little study has been done on this aspect for discrete systems. Section 4 is devoted to the main results of this paper, the proposed system is investigated as it undergoes a Neimark-Sacker bifurcation and a flip bifurcation. Section 5 contains a numerical verification of some key results. Conclusion and discussion are presented in Sect. 6.

\section{Statement of model and local parameterization}

Consider the scaled equation, describing a singular bioeconomic system (see [6, 9, 17-19]) with nonlinear harvesting function, as

$$
\left\{\begin{array}{l}
\dot{x}=x(a-k x-y)-\frac{x E}{1+m x}, \\
\dot{y}=y(-s+x), \\
v=\frac{p x E}{1+m x}-c E .
\end{array}\right.
$$

For the practical significance, the system (2.1) is defined on the set

$$
R_{+}^{3}=\left\{(x, y, E) \in R_{+}^{3} \mid x \geq 0, y \geq 0, E \geq 0\right\},
$$

and is subject to positive initial conditions,

$$
x(0)>0, \quad y(0)>0, \quad E(0)>0 .
$$

Equation (2.1) is a semi-explicit autonomous differential-algebraic equations with differential index $1, \frac{x E}{1+m x}$ is the harvesting function with constant $m$ being related to the handling or processing time $[17,19]$. Though this nonlinear harvesting function is more realistic, not many researchers have used it in their studies. For $m=0$ it reduces to the Schaefer catch-effort equation (1.2).

We put $(x, y, E)^{T} \triangleq X$ and use $X_{0}=\left(x_{0}, y_{0}, E_{0}\right)^{T}$ to denote the equilibria of (2.1). It can be checked that system (2.1) has a unique equilibria,

$$
X_{0}=\left(s, a-k s-\frac{v}{(p-c m) s-c}, \frac{(1+m s) v}{(p-c m) s-c}\right)^{T} .
$$


We have $X_{0} \in R_{+}^{3}$, in this work, we take parameters $(a, k, s, p, m, c, v)$ from the set

$$
\pi=\left\{(a, k, s, p, m, c, v) \mid a-k s-\frac{v}{(p-c m) s-c}>0,(p-c m) s-c>0\right\} .
$$

For (2.1), if derivatives are replaced by finite difference approximations, then we get the forward Euler difference scheme with step-size $\delta$ :

$$
\left\{\begin{array}{l}
x_{n+1}=x_{n}+\delta x_{n}\left(a-k x_{n}-y_{n}-\frac{E_{n}}{1+m x_{n}}\right), \\
y_{n+1}=y_{n}+\delta y_{n}\left(-s+x_{n}\right), \\
0=\frac{p x_{n} E_{n}}{1+m x_{n}}-c E_{n}-v,
\end{array}\right.
$$

or in map form

$$
\left\{\begin{array}{l}
x \mapsto x+\delta x\left(a-k x-y-\frac{E}{1+m x}\right) \\
y \mapsto y+\delta y(-s+x) \\
0=\frac{p x E}{1+m x}-c E-v
\end{array}\right.
$$

We can easily verify that the fixed point of (2.3) is also $X_{0}$. Now we will determine the local equivalent parameterization system of (2.3).

We first rewrite (2.1) into the constrained form [16], that is, the differential equations on the manifold,

$$
\left\{\begin{array}{l}
\dot{x}=x(a-k x-y)-\frac{x E}{1+m x} \\
\dot{y}=y(-s+x) \\
\dot{E}=\delta \tilde{f}_{3}(x, y, E) \\
0=\frac{p x E}{1+m x}-c E-v
\end{array}\right.
$$

The algebraic equation sets an invariant manifold (denoted by $M$ ) in $R^{3}$ [16] whose number of independent coordinates is $2 . \dot{E}$ is described through a derivation of the algebraic equation along the solution path with respect to time $t$ and it is not necessary for the concrete formula as we will see later. Accordingly, (2.2) becomes the following difference algebraic equations of the constrained form:

$$
\left\{\begin{array}{l}
x_{n+1}=x_{n}+\delta x_{n}\left(a-k x_{n}-y_{n}-\frac{E_{n}}{1+m x_{n}}\right), \\
y_{n+1}=y_{n}+\delta y_{n}\left(-s+x_{n}\right), \\
E_{n+1}=E_{n}+\delta \tilde{f}_{3}\left(x_{n}, y_{n}, E_{n}\right), \\
0=\frac{p x_{n} E_{n}}{1+m x_{n}}-c E_{n}-v .
\end{array}\right.
$$

It will be convenient to introduce some notation:

$$
\begin{aligned}
& f_{1}(x, y, E)=x+\delta x\left(a-k x-y-\frac{E}{1+m x}\right), \\
& f_{2}(x, y, E)=y+\delta y(-s+x)
\end{aligned}
$$




$$
\begin{aligned}
& f_{3}(x, y, E)=E+\delta \tilde{f}_{3}(x, y, E), \\
& g(x, y, E)=\frac{p x E}{1+m x}-c E-v,
\end{aligned}
$$

and the vector-valued function

$$
f=\left(f_{1}, f_{2}\right)^{T}, \quad \tilde{f}=\left(f_{1}, f_{2}, f_{3}\right)^{T} .
$$

In terms of the above notations, (2.5) becomes

$$
\left\{\begin{array}{l}
X_{n+1}=\tilde{f}\left(X_{n}\right), \\
g\left(X_{n}\right)=0,
\end{array}\right.
$$

or in map form

$$
\left\{\begin{array}{l}
X \mapsto \tilde{f}(X), \\
g(X)=0 .
\end{array}\right.
$$

Note that the form of (2.6) or (2.7) is generic (representing difference algebraic equations in constrained form). We next generalize the tangent space local parameterization method for general differential-algebraic equations in constrained form to parameterize (2.6) or (2.7). First we give a decomposition for $M$ in $R^{3}$ as follows:

$$
X-X_{0}=U_{0} Y+V_{0} \tilde{Y}, \quad \forall X \in M,
$$

or

$$
X=X_{0}+U_{0} Y+V_{0} \tilde{Y}, \quad \forall X \in M
$$

$\left[U_{0}, V_{0}\right]$ is an arbitrary order-three orthogonal matrix whose columns consist of a basis for $R^{3}$ with $U_{0}$ is $3 \times 2$ and $V_{0}$ is $3 \times 1 . Y:=\left(y_{1}, y_{2}\right)^{T} \in R^{2}$ is the coordinate associated with the projection of $X-X_{0}$ onto the column space of $U_{0}$, and $\tilde{Y} \in R$ is the coordinate associated with the projection of $X-X_{0}$ onto the column space of $V_{0}$; note that $\tilde{Y}$ might be locally represented by $Y$. Indeed,

$$
g\left(X_{0}+U_{0} Y+V_{0} \tilde{Y}\right)=0
$$

Let $G(Y, \widetilde{Y})=g\left(x_{0}+U_{0} Y+V_{0} \tilde{Y}\right)$, therefore

$$
\operatorname{det}\left(D_{\widetilde{Y}} G(Y, \widetilde{Y})\right)=D_{\widetilde{Y}} g\left(X_{0}+U_{0} Y+V_{0} \widetilde{Y}\right)=D g \cdot V_{0} \neq 0,
$$

where $D$ is a derivation operator. Hence, there exists a smooth map: $h: R^{2} \rightarrow R$ guaranteed by the implicit function theorem such that $\widetilde{Y}=h(Y)$ locally holds. Putting it in (2.8) gives a local parameterization of the solution manifold with parameter $Y$ as follows:

$$
X=X_{0}+U_{0} Y+V_{0} h(Y) \equiv \psi(Y)
$$


where

$$
\psi(Y)=\left(\begin{array}{l}
\psi_{1}\left(y_{1}, y_{2}\right) \\
\psi_{2}\left(y_{1}, y_{2}\right) \\
\psi_{3}\left(y_{1}, y_{2}\right)
\end{array}\right) .
$$

Obviously, $\psi$ is related to $h$.

From (2.9) together with the first equation of system (2.6) we get

$$
\psi\left(Y_{n+1}\right)=\tilde{f}\left(\psi\left(Y_{n}\right)\right),
$$

which gives

$$
X_{0}+U_{0} Y_{n+1}+V_{0} h\left(Y_{n+1}\right)=\tilde{f}\left(\psi\left(Y_{n}\right)\right)
$$

multiplying by $U_{0}^{T}$ gives

$$
U_{0}^{T} X_{0}+Y_{n+1}=U_{0}^{T} \tilde{f}\left(\psi\left(Y_{n}\right)\right),
$$

that is,

$$
Y_{n+1}=U_{0}^{T} \tilde{f}\left(\psi\left(Y_{n}\right)\right)-U_{0}^{T} X_{0}
$$

or in map form

$$
Y \mapsto U_{0}^{T} \tilde{f}(\psi(Y))-U_{0}^{T} X_{0},
$$

which is the local parameterization of (2.7) near $X_{0}$ with parameter $Y$.

Specifically, if take

$$
U_{0}=\left(\begin{array}{ll}
1 & 0 \\
0 & 1 \\
0 & 0
\end{array}\right), \quad V_{0}=\left(\begin{array}{l}
0 \\
0 \\
1
\end{array}\right)
$$

then

$$
U_{0}^{T} \tilde{f}\left(\psi\left(Y_{n}\right)\right)=f\left(\psi\left(Y_{n}\right)\right) .
$$

Here we see that $\tilde{f}_{3}(X)$ vanishes, which is why it is not necessary to give the concrete formula of $\tilde{f}_{3}$ in $\dot{E}$ of the equation of (2.4). Hence using (2.10) and (2.11), we obtain the following parameterization system:

$$
Y_{n+1}=f\left(\psi\left(Y_{n}\right)\right)-U_{0}^{T} X_{0},
$$

or in map form

$$
Y \mapsto f(\psi(Y))-U_{0}^{T} X_{0},
$$


that is,

$$
Y \mapsto f(\psi(Y))-\left(x_{0}, y_{0}\right)^{T}
$$

Equation (2.12) is topologically equivalent, near the origin, to the difference algebraic system (2.3) in a neighborhood of $X_{0}$.

Remark 2.1 The fixed point $X_{0}$ of (2.3) corresponds to the fixed point $O(0,0)$ of $(2.12)$.

Remark 2.2 Since the form of (2.6) or (2.7) has generality, the new parameterization method is also generic ( $X_{n}$ can be of arbitrary finite dimensions). Specifically, if we take the tangent space and normal vector space of solution manifold at $X_{0}$ as $U_{0}$ and $V_{0}$, respectively, then the local parameterization (2.9) reduces to the tangent space local parameterization condensed method for DAEs in [16].

\section{Fixed points classification and stability analysis}

Our goal in this section is to examine the local stability of (2.3) based upon the standard linearization technique.

From $g(\psi(Y))=0$ we obtain

$$
D g(X) D \psi(Y)=0 .
$$

Differentiating (2.9) with respect to $y$ and multiplying both sides by $U_{0}^{T}$ gives

$$
U_{0}^{T} D \psi(Y)=I_{2}
$$

Combine (3.1) and (3.2) to yield

$$
\begin{aligned}
D \psi(Y) & =\left(\begin{array}{c}
D g(X) \\
\left.U_{0}^{T}\right)^{-1}
\end{array}\left(\begin{array}{l}
0 \\
I_{2}
\end{array}\right)\right. \\
& =\left(\begin{array}{cc}
1 & 0 \\
0 & 1 \\
-\frac{E p}{((p-c m) x-c)(1+m x)} & 0
\end{array}\right)
\end{aligned}
$$

for future use. Note that $D \psi(Y)$ has nothing to do with $h$, so we can give the concrete form of (2.12) by a Taylor formula.

The Jacobian matrix of the system (2.12) evaluated at the origin is given by

$$
\begin{aligned}
D & \left.\left(f(\psi(y))-\left(x_{0}, y_{0}\right)^{T}\right)\right|_{(0,0)} \\
& =\left.\left.D f(X)\right|_{X_{0}} D \psi(Y)\right|_{(0,0)} \\
& =\left.\left(\begin{array}{lll}
f_{1 x} & f_{1 y} & f_{1 E} \\
f_{2 x} & f_{2 y} & f_{2 E}
\end{array}\right)\right|_{X_{0}}\left(\begin{array}{cc}
1 & 0 \\
0 & 1 \\
-\frac{E_{0} p}{((p-c m) s-c)(1+m s)} & 0
\end{array}\right) \\
& =\left(\begin{array}{cc}
1-\delta s\left(k-\frac{v m}{((p-c m) s-c)(1+m s)}\right)+\frac{v}{((p-c m) s-c)^{2}(1+m s)} & -\delta s \\
\delta\left(a-k s-\frac{v}{(p-c m) s-c}\right) & 1
\end{array}\right) .
\end{aligned}
$$


Hence the characteristic equation associated to the Jacobian matrix is given by

$$
F(\lambda)=\lambda^{2}+B \lambda+C=0,
$$

where

$$
\left\{\begin{aligned}
B= & \delta s\left(k-\frac{v m}{((p-c m) s-c)(1+m s)}\right)-\frac{p \delta s v}{((p-c m) s-c)^{2}(1+m s)}-2 \\
C= & +\frac{p \delta s v}{((p-c m) s-c)^{2}(1+m s)}-\delta s\left(k-\frac{v m}{((p-c m) s-c)(1+m s)}\right) \\
& +s \delta^{2}\left(a-k s-\frac{v}{(p-c m) s-c}\right) .
\end{aligned}\right.
$$

Putting $\lambda=1$ and $\lambda=-1$ in $F(\lambda)$, respectively, we get

$$
F(1)=s \delta^{2} y_{0}=s \delta^{2}\left(a-k s-\frac{v}{(p-c m) s-c}\right)
$$

and

$$
\begin{aligned}
F(-1)= & 4+\frac{2 E_{0} p \delta s}{((p-c m) s-c)(1+m s)^{2}}-2 \delta s\left(k-\frac{E_{0} m}{(1+m s)^{2}}\right)+\delta^{2} s y_{0} \\
= & 4+\frac{2 p \delta s v}{((p-c m) s-c)^{2}(1+m s)}-2 \delta s\left(k-\frac{v m}{((p-c m) s-c)(1+m s)}\right) \\
& +s \delta^{2}\left(a-k s-\frac{v}{(p-c m) s-c}\right) .
\end{aligned}
$$

Let $\lambda_{1}$ and $\lambda_{2}$ denote the two roots of (3.3), referred to as the eigenvalues (or multipliers) of the fixed point $O(0,0)$. We first recall the relevant definitions of topological types for fixed points (see [20]).

Definition 3.1 $O(0,0)$ is called

(i) a hyperbolic fixed point, if the moduli of all eigenvalues do not equal 1; and

(ii) a nonhyperbolic fixed point, if $\left|\lambda_{1}\right|=1$ or $\left|\lambda_{2}\right|=1$.

Definition 3.2 If $O(0,0)$ is a hyperbolic fixed point, then it is called

(i) a sink, if $\left|\lambda_{1}\right|<1$ and $\left|\lambda_{2}\right|<1$;

(ii) a source, if $\left|\lambda_{1}\right|>1$ and $\left|\lambda_{2}\right|>1$; and

(iii) a saddle, if $\lambda_{1,2}$ are real, with $\left|\lambda_{1}\right|<1$ and $\left|\lambda_{2}\right|>1$ (or $\left|\lambda_{1}\right|>1$ and $\left|\lambda_{2}\right|<1$ ).

Moreover, we can classify fixed points based on the geometric properties of trajectory distribution near them.

Definition 3.3 $O(0,0)$ is called

(i) a saddle, if $\lambda_{1,2}$ are real, with $\left|\lambda_{1}\right|<1$ and $\left|\lambda_{2}\right|>1$ (or $\left|\lambda_{1}\right|>1$ and $\left|\lambda_{2}\right|<1$ );

(ii) a node, if $\lambda_{1,2}$ are real, with $\left|\lambda_{1,2}\right|<1$ (or $\left|\lambda_{1,2}\right|>1$ );

(iii) a focus (sometimes called spiral point), if $\lambda_{1,2}=r(\cos \theta \pm i \sin \theta), r \neq 1$; and

(iv) a center, if $\lambda_{1,2}=\cos \theta \pm i \sin \theta$.

Thus, we have the following lemma from the relations between roots and coefficients of the quadratic equation [21]. 
Lemma 3.1 Suppose that $F(1)>0$, then we have

(i) $\left|\lambda_{1}\right|<1$ and $\left|\lambda_{2}\right|<1$ iff $F(-1)>0$ and $C<1$;

(ii) $\left|\lambda_{1}\right|>1$ and $\left|\lambda_{2}\right|>1$ iff $F(-1)>0$ and $C>1$;

(iii) $\left|\lambda_{1}\right|<1$ and $\left|\lambda_{2}\right|>1$ (or $\left|\lambda_{1}\right|>1$ and $\left.\left|\lambda_{2}\right|<1\right)$ iff $F(-1)<0$;

(iv) $\lambda_{1}$ and $\lambda_{2}$ are complex and $\left|\lambda_{1}\right|=\left|\lambda_{2}\right|=1$ iff $B^{2}-4 C<0$ and $C=1$; and

(v) $\lambda_{1}=-1$ and $\left|\lambda_{2}\right| \neq 1$ iff $F(-1)=0$ and $B \neq 0,2$.

Using Lemma 3.1, we obtain the following results.

Theorem 3.1 Assuming parameters ( $a, k, s, p, m, c, v) \in \pi$, system (2.3) has a unique positive fixed point $X_{0}$ and

(i) it is a sink iff

$$
4+2 \delta \Lambda_{1}-2 \delta \Lambda_{2}+\delta^{2} \Lambda_{3}>0 \text { and } \Lambda_{1}-\Lambda_{2}+\delta \Lambda_{3}<0
$$

(ii) it is a source iff

$$
4+2 \delta \Lambda_{1}-2 \delta \Lambda_{2}+\delta^{2} \Lambda_{3}>0 \text { and } \Lambda_{1}-\Lambda_{2}+\delta \Lambda_{3}>0
$$

(iii) it is a saddle iff

$$
4+2 \delta \Lambda_{1}-2 \delta \Lambda_{2}+\delta^{2} \Lambda_{3}<0 ; \quad \text { and }
$$

(iv) it is nonhyperbolic if one of the following conditions is satisfied:

(iv1) $\delta \Lambda_{3}=\Lambda_{2}-\Lambda_{1}$ and $\left(\Lambda_{2}-\Lambda_{1}\right)^{2}-4 \Lambda_{3}<0$;

(iv2) $4+2 \delta \Lambda_{1}-2 \delta \Lambda_{2}+\delta^{2} \Lambda_{3}=0$ and $\delta \Lambda_{2}-\delta \Lambda_{1} \neq 2$, 4 .

Theorem 3.2 With the assumption of Theorem 3.1, $X_{0}$ is

(i) a stable node iff

$$
\delta\left(\Lambda_{2}-\Lambda_{1}\right)<2 \text { and } 1+\delta \Lambda_{1}-\delta \Lambda_{2}+\delta^{2} \Lambda_{3}>0 ;
$$

(ii) an unstable node iff

$$
\Lambda_{2}-\Lambda_{1}<0
$$

(iii) a stable focus iff

$$
\left(\Lambda_{2}-\Lambda_{1}\right)^{2}-4 \Lambda_{3}<0 \text { and } \delta \Lambda_{3}<\Lambda_{2}-\Lambda_{1}
$$

(iv) an unstable focus iff

$$
\left(\Lambda_{2}-\Lambda_{1}\right)^{2}-4 \Lambda_{3}<0 \text { and } \delta \Lambda_{3}>\Lambda_{2}-\Lambda_{1}
$$

where

$$
\Lambda_{1}=\frac{p s v}{((p-c m) s-c)^{2}(1+m s)},
$$




$$
\begin{aligned}
& \Lambda_{2}=s\left(k-\frac{v m}{((p-c m) s-c)(1+m s)}\right), \\
& \Lambda_{3}=s\left(a-k s-\frac{v}{(p-c m) s-c}\right) .
\end{aligned}
$$

\section{Bifurcation analysis}

Based on the analysis of Sect. 3, we discuss a codimension-one bifurcation (NeimarkSacker bifurcation and flip bifurcation) of system (2.3) at the fixed point.

\subsection{Neimark-Sacker bifurcation and invariant curve}

First, let $C=1$, then we have

$$
\begin{aligned}
& s\left(a-k s-\frac{v}{(p-c m) s-c}\right) \delta^{2}+\frac{p s v \delta}{((p-c m) s-c)^{2}(1+m s)} \\
& -s\left(k-\frac{v m}{((p-c m) s-c)(1+m s)}\right) \delta=0 .
\end{aligned}
$$

Solving this equation gives

$$
\delta_{1}=\frac{\Lambda_{2}-\Lambda_{1}}{\Lambda_{3}}
$$

We choose $\delta$ as a bifurcation parameter to discuss the Neimark-Sacker bifurcation when $\delta$ varies in a small neighborhood of $\delta_{1}$. If we consider $\delta^{*}=\delta-\delta_{1}$, then $\delta=\delta_{1}$ is equivalent to $\delta^{*}=0$.

The linearization of (2.12) has the characteristic equation at $O(0,0)$

$$
F(\lambda)=\lambda^{2}+B\left(\delta^{*}\right) \lambda+C\left(\delta^{*}\right)=0,
$$

where

$$
\left\{\begin{aligned}
B\left(\delta^{*}\right)= & \left(\delta^{*}+\delta_{1}\right) s\left(k-\frac{v m}{((p-c m) s-c)(1+m s)}\right)-\frac{\left(\delta^{*}+\delta_{1}\right) p s v}{((p-c m) s-c)^{2}(1+m s)}-2 \\
C\left(\delta^{*}\right)=1 & +\frac{\left(\delta^{*}+\delta_{1}\right) p s v}{((p-c m) s-c)^{2}(1+m s)}-\left(\delta^{*}+\delta_{1}\right) s\left(k-\frac{v m}{((p-c m) s-c)(1+m s)}\right) \\
& +s\left(\delta^{*}+\delta_{1}\right)^{2}\left(a-k s-\frac{v}{(p-c m) s-c}\right) .
\end{aligned}\right.
$$

Let

$$
\Omega_{1}=\left\{(a, k, s, p, m, c, v, \delta) \mid(a, k, s, p, m, c, v) \in \pi, 0<\delta=\delta_{1},\left(\Lambda_{2}-\Lambda_{1}\right)^{2}<4 \Lambda_{3}\right\} .
$$

Then, if $(k, a, m, s, p, c, v, \delta) \in \Omega_{1}$, the characteristic values are complex conjugate numbers $\lambda$ and $\bar{\lambda}$ with $|\lambda|=|\bar{\lambda}|=1$, where

$$
\lambda, \bar{\lambda}=-\frac{B\left(\delta^{*}\right)}{2} \pm \frac{i}{2} \sqrt{4 C\left(\delta^{*}\right)-B^{2}\left(\delta^{*}\right)}, \quad|\lambda|_{\delta^{*}=0}=\sqrt{C(0)}=1
$$

and

$$
\alpha=\left.\frac{d\left|\lambda\left(\delta^{*}\right)\right|}{d \delta^{*}}\right|_{\delta^{*}=0}=\frac{1}{2} \frac{\Lambda_{2}-\Lambda_{1}}{\sqrt{1+\Lambda_{1} \delta_{1}-\Lambda_{2} \delta_{1}+\Lambda_{3} \delta_{1}^{2}}}=\frac{\Lambda_{2}-\Lambda_{1}}{2}>0 .
$$


In addition, it is required that when $\delta^{*}=0, \lambda^{m}, \bar{\lambda}^{m} \neq 1(m=1,2,3,4)$, which is equivalent to $B(0) \neq-2,0,1,2$, that is, $\delta_{1} \Lambda_{2}-\delta_{1} \Lambda_{1} \neq 0,2,3,4$. Note that $\Lambda_{2}>\Lambda_{1}$, thus

$$
H=\delta_{1} \Lambda_{2}-\delta_{1} \Lambda_{1} \neq 2,3,4
$$

If we put

$$
\begin{aligned}
& x+\left(\delta^{*}+\delta_{1}\right) x(a-k x-y)-\frac{x E}{1+m x}-x_{0} \triangleq F_{1}(X), \\
& y+\left(\delta^{*}+\delta_{1}\right) y(-s+x)-y_{0} \triangleq F_{2}(X),
\end{aligned}
$$

and

$$
\left(F_{1}, F_{2}\right)^{T} \triangleq F_{\delta^{*}},
$$

then

$$
Y \mapsto F(\psi(Y))-\left(x_{0}, y_{0}\right)^{T}
$$

is a one-parameter map with parameter $\delta^{*}$.

We next determine, in order to get the normal form of (4.2), the Taylor expansion of the right-hand side of (4.2) at the origin to order three, that is,

$$
\left(\begin{array}{l}
y_{1} \\
y_{2}
\end{array}\right) \mapsto\left(\begin{array}{c}
a_{1} y_{1}+a_{2} y_{2}+a_{11} y_{1}^{2}+a_{12} y_{1} y_{2}+a_{111} y_{1}^{3}+O\left(\left(\left|y_{1}\right|+\left|y_{2}\right|\right)^{4}\right) \\
b_{1} y_{1}+b_{2} y_{2}+b_{12} y_{1} y_{2}+O\left(\left(\left|y_{1}\right|+\left|y_{2}\right|\right)^{4}\right)
\end{array}\right)
$$

where the terms with 0 coefficients are omitted. By the chain rule, we have the following:

$$
\left\{\begin{aligned}
a_{1}= & \left.\frac{\partial F_{1}(\psi(Y))}{\partial y_{1}}\right|_{(0,0)}=1+\left(\delta^{*}+\delta_{1}\right) s\left(-k+\frac{E_{0} m}{(1+m s)^{2}}+\frac{E_{0} p}{(1+m s)^{2}((p-c m) s-c)}\right), \\
a_{2}= & \left.\frac{\partial F_{1}(\psi(Y))}{\partial y_{2}}\right|_{(0,0)}=-\left(\delta^{*}+\delta_{1}\right) s, \\
a_{11}= & \left.\frac{\partial^{2} F_{1}(\psi(Y))}{\partial y_{1}^{2}}\right|_{(0,0)} \\
= & \frac{\left(-c^{2} k m^{3} s^{3}+2 c k m^{2} p s^{3}-3 c^{2} k m^{2} s^{2}-k m p^{2} s^{3}\right)\left(\delta_{1}+\delta^{*}\right)}{(c m s-p s+c)^{2}(m s+1)} \\
& \quad+\frac{\left(4 c k m p s^{2}-3 c^{2} k m s-k p^{2} s^{2}+c^{2} E_{0} m\right)\left(\delta_{1}+\delta^{*}\right)}{(c m s-p s+c)^{2}(m s+1)} \\
& \quad+\frac{\left(2 c k p s-c^{2} k-c E_{0} p\right)\left(\delta_{1}+\delta^{*}\right)}{(c m s-p s+c)^{2}(m s+1)}, \\
a_{12}= & \left.\frac{\partial^{2} F_{1}(\psi(Y))}{\left.\partial y_{1} \partial y_{2}\right)}\right|_{(0,0)}=-\delta^{*}-\delta_{1}, \\
a_{111}= & \left.\frac{\partial^{3} F_{1}(\psi(Y))}{\partial y_{1}^{3}}\right|_{(0,0)}=-\frac{\left(c^{2} m^{2}-2 c m p+p^{2}\right) E_{0} c\left(\delta^{*}+\delta_{1}\right)}{(c m s-p s+c)^{3}(1+m s)}, \\
b_{1}= & \left.\frac{\partial F_{2}(\psi(Y))}{\partial y_{1}}\right|_{(0,0)}=\left(\delta^{*}+\delta_{1}\right) y_{0}, \\
b_{2}= & \left.\frac{\partial F_{2}(\psi(Y))}{\partial y_{2}}\right|_{(0,0)}=1, \quad b_{12}=\left.\frac{\partial^{2} F_{2}(\psi(Y))}{\partial y_{1} \partial y_{2}}\right|_{(0,0)}=\delta^{*}+\delta_{1} .
\end{aligned}\right.
$$

Let

$$
\sigma=-\frac{B(0)}{2}, \quad \omega=\frac{\sqrt{4 C(0)-B^{2}(0)}}{2},
$$


then through the change of basis with

$$
\begin{aligned}
& \left(\begin{array}{l}
y_{1} \\
y_{2}
\end{array}\right)=T\left(\begin{array}{l}
z_{1} \\
z_{2}
\end{array}\right), \\
& T=\left(\begin{array}{cc}
a_{2} & 0 \\
\sigma-a_{1} & -\omega
\end{array}\right), \\
& T^{-1}=\left(\begin{array}{cc}
\frac{1}{a_{2}} & 0 \\
\frac{-\sigma+a_{1}}{a_{2} \omega} & -\frac{1}{\omega}
\end{array}\right),
\end{aligned}
$$

we can change system (4.3) into the normal form

$$
\left(\begin{array}{l}
z_{1} \\
z_{2}
\end{array}\right) \mapsto\left(\begin{array}{cc}
\sigma & -\omega \\
\omega & \sigma
\end{array}\right)\left(\begin{array}{l}
z_{1} \\
z_{2}
\end{array}\right)+\left(\begin{array}{l}
\bar{f}_{1}\left(z_{1}, z_{2}\right) \\
\bar{f}_{1}\left(z_{1}, z_{2}\right)
\end{array}\right)
$$

where the nonlinear terms are

$$
\begin{aligned}
\bar{f}_{1}\left(z_{1}, z_{2}\right)= & \left(a_{2} a_{11}+a_{12} \sigma-a_{12} a_{1}\right) z_{1}^{2}-a_{12} \omega z_{1} z_{2}+a_{111} a_{2}^{2} z_{1}^{3}+O\left(\left(\left|z_{1}\right|+\left|z_{2}\right|\right)^{4}\right), \\
\bar{f}_{2}\left(z_{1}, z_{2}\right)= & \frac{\left(\sigma-a_{1}\right)\left(a_{2} a_{11}-a_{1} a_{12}+a_{12} \sigma\right)-a_{2} b_{12}\left(\sigma-a_{1}\right)}{\omega} z_{1}^{2} \\
& +\left(a_{2} b_{12}-\frac{\left(\sigma-a_{1}\right) a_{12} \omega}{\omega}\right) z_{1} z_{2} \\
& +\frac{\left(\sigma-a_{1}\right) a_{111} a_{2}^{2}}{\omega} z_{1}^{3}+O\left(\left(\left|z_{1}\right|+\left|z_{2}\right|\right)^{4}\right),
\end{aligned}
$$

together with

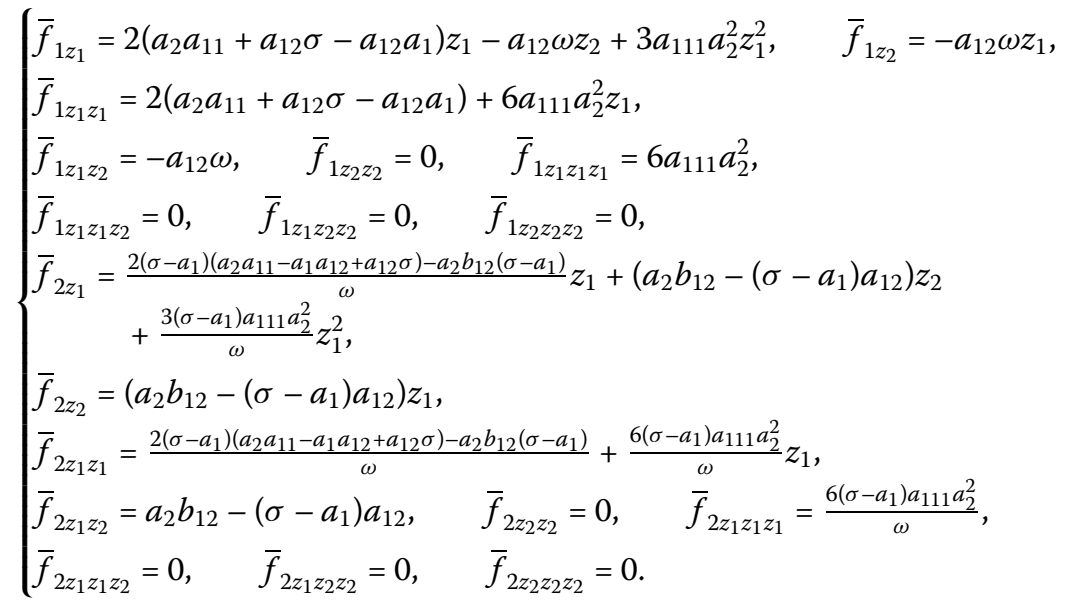

In order for system (4.4) to undergo a Neimark-Sacker bifurcation at $O(0,0)$, we require that the following quantity is not zero [22]:

$$
l=-\operatorname{Re}\left(\frac{(1-2 \lambda) \bar{\lambda}^{2}}{1-\lambda} \gamma_{20} \gamma_{11}\right)-\frac{1}{2}\left|\gamma_{11}\right|^{2}-\left|\gamma_{02}\right|^{2}+\operatorname{Re}\left(\bar{\lambda} \gamma_{21}\right),
$$


where

$$
\begin{aligned}
& \gamma_{20}=\frac{\left(\bar{f}_{1 z_{1} z_{1}}-\bar{f}_{1 z_{2} z_{2}}+2 \bar{f}_{2 z_{1} z_{2}}\right)+i\left(\bar{f}_{2 z_{1} z_{1}}-\bar{f}_{2 z_{2} z_{2}}-2 \bar{f}_{1 z_{1} z_{2}}\right)}{8}, \\
& \gamma_{11}=\frac{\left(\bar{f}_{1 z_{1} z_{1}}+\bar{f}_{1 z_{2} z_{2}}\right)+i\left(\bar{f}_{2 z_{1} z_{1}}+\bar{f}_{2 z_{2} z_{2}}\right)}{4}, \\
& \gamma_{02}=\frac{\left(\bar{f}_{1 z_{1} z_{1}}-\bar{f}_{1 z_{2} z_{2}}-2 \bar{f}_{2 z_{1} z_{2}}\right)+i\left(\bar{f}_{2 z_{1} z_{1}}-\bar{f}_{2 z_{2} z_{2}}+2 \bar{f}_{1 z_{1} z_{2}}\right)}{8}, \\
& \gamma_{21}=\frac{\left(\bar{f}_{1 z_{1} z_{1} z_{1}}+\bar{f}_{1 z_{1} z_{2} z_{2}}+\bar{f}_{2 z_{1} z_{1} z_{2}}+\bar{f}_{2 z_{2} z_{2} z_{2}}\right)+i\left(\bar{f}_{2 z_{1} z_{1} z_{1}}+\bar{f}_{2 z_{1} z_{2} z_{2}}-\bar{f}_{1 z_{1} z_{1} z_{2}}-\bar{f}_{1 z_{2} z_{2} z_{2}}\right)}{16} .
\end{aligned}
$$

Note that here all the derivatives are taken at $O(0,0)$. From the above discussion, we obtain the following result.

Theorem 4.1 Assume that ( $a, k, s, p, m, c, v, \delta) \in \Omega_{1}$ and the condition (4.1) holds. If $l \neq 0$, then the system (2.3) undergoes a Neimark-Sacker bifurcation at the fixed point $\left(x_{0}, y_{0}, E_{0}\right)$ when the bifurcation parameter $\delta$ varies in a small neighborhood of $\delta_{1}$. Moreover, if $l<0$ (resp. $l>0$ ), then an attracting (resp. repelling) invariant closed curve bifurcates from the fixed point for $\delta>\delta_{1}\left(\right.$ resp. $\left.\delta<\delta_{1}\right)$.

\subsection{Flip bifurcation and chaos}

We proceed to investigate the flip bifurcation. For (3.3), let $F(-1)=0$ we obtain

$$
\begin{aligned}
4 & +\frac{2 p \delta s v}{((p-c m) s-c)^{2}(1+m s)}-2 \delta s\left(k-\frac{v m}{((p-c m) s-c)(1+m s)}\right) \\
& +s \delta^{2}\left(a-k s-\frac{v}{(p-c m) s-c}\right)=0 .
\end{aligned}
$$

Solving this equation gives

$$
\delta_{2}=\frac{\Lambda_{2}-\Lambda_{1}-\sqrt{\left(\Lambda_{2}-\Lambda_{1}\right)^{2}-4 \Lambda_{3}}}{\Lambda_{3}} .
$$

Here we still choose $\delta$ as a bifurcation parameter to discuss the flip bifurcation when $\delta$ varies in a small neighborhood of $\delta_{2}$. In the same way as above, we consider $\delta^{*}=\delta-\delta_{2}$, then $\delta=\delta_{2}$ is equivalent to $\delta^{*}=0$. Since we would like to study the system as we vary $\delta^{*}$ near 0 , we treat $\delta^{*}$ as a variable (which has no dependence on time) and hence the parameterfree center manifold theorem can easily be extended for a parameter-dependent system.

We begin by putting

$$
\bar{X}=\left(x, y, E, \delta^{*}\right)^{T}, \quad \bar{Y}=\left(y_{1}, y_{2}, \delta^{*}\right)^{T} .
$$

Accordingly, the local parameterization (2.9) should be rewritten as

$$
\bar{X}=\widetilde{\psi}(\bar{Y}),
$$


where

$$
\begin{aligned}
\widetilde{\psi}(\bar{Y}) & =\widetilde{\psi}\left(y_{1}, y_{2}, \delta^{*}\right) \\
& =\left(\begin{array}{c}
\widetilde{\psi}_{1}\left(y_{1}, y_{2}, \delta^{*}\right) \\
\widetilde{\psi}_{2}\left(y_{1}, y_{2}, \delta^{*}\right) \\
\widetilde{\psi}_{3}\left(y_{1}, y_{2}, \delta^{*}\right) \\
\widetilde{\psi}_{0}\left(y_{1}, y_{2}, \delta^{*}\right)
\end{array}\right),
\end{aligned}
$$

together with

$$
\begin{aligned}
& \psi_{1}\left(y_{1}, y_{2}\right) \triangleq \widetilde{\psi}_{1}\left(y_{1}, y_{2}, \delta^{*}\right), \\
& \psi_{2}\left(y_{1}, y_{2}\right) \triangleq \widetilde{\psi}_{2}\left(y_{1}, y_{2}, \delta^{*}\right), \\
& \psi_{3}\left(y_{1}, y_{2}\right) \triangleq \widetilde{\psi}_{3}\left(y_{1}, y_{2}, \delta^{*}\right),
\end{aligned}
$$

and

$$
\delta^{*} \triangleq \widetilde{\psi}_{0}\left(y_{1}, y_{2}, \delta^{*}\right) .
$$

We put

$$
F_{1}(\bar{X})=x+\left(\delta^{*}+\delta_{2}\right) x(a-k x-y)-\frac{x E}{1+m x}-x_{0}
$$

and

$$
F_{2}(\bar{X})=y+\left(\delta^{*}+\delta_{2}\right) y(-s+x)-y_{0}, \quad \text { and } \quad F=\left(F_{1}, F_{2}\right)^{T} .
$$

Hence we study the three-dimensional extended system

$$
\bar{Y} \mapsto\left(\begin{array}{c}
F(\widetilde{\psi}(\bar{Y}))-\left(x_{0}, y_{0}\right)^{T} \\
\delta^{*}
\end{array}\right) .
$$

As in our study of the Neimark-Sacker bifurcation, we expand the right-hand side of (4.5) in the order-three Taylor polynomial,

$$
\left(\begin{array}{l}
y_{1} \\
y_{2} \\
\delta^{*}
\end{array}\right) \mapsto\left(\begin{array}{c}
a_{1} y_{1}+a_{2} y_{2}+a_{11} y_{1}^{2}+a_{12} y_{1} y_{2}+a_{13} y_{1} \delta^{*}+a_{23} y_{2} \delta^{*}+a_{111} y_{1}^{3} \\
+a_{113} y_{1}^{2} \delta^{*}+a_{123} y_{1} y_{2} \delta^{*}+O\left(\left(\left|y_{1}\right|+\left|y_{2}\right|+\left|\delta^{*}\right|\right)^{4}\right) \\
b_{1} y_{1}+b_{2} y_{2}+b_{12} y_{1} y_{2}+b_{13} y_{1} \delta^{*}+b_{123} y_{1} y_{2} \delta^{*}+O\left(\left(\left|y_{1}\right|+\left|y_{2}\right|+\left|\delta^{*}\right|\right)^{4}\right) \\
\delta^{*}
\end{array}\right)
$$


where

$$
\left\{\begin{aligned}
a_{1}= & \left.\frac{\partial F_{1}(\widetilde{\psi}(\bar{Y}))}{\partial y_{1}}\right|_{(0,0,0)}=1+\delta_{2} s\left(-k+\frac{E_{0} m}{(1+m s)^{2}}\right)+\frac{\delta_{2} s E_{0} p}{(1+m s)^{2}((p-c m) s-c)}, \\
a_{2}= & \left.\frac{\partial F_{1}(\widetilde{\psi}(\bar{Y}))}{\partial y_{2}}\right|_{(0,0,0)}=-\delta_{2} s, \quad a_{12}=\left.\frac{\partial^{2} F_{1}(\widetilde{\psi}(\bar{Y}))}{\partial y_{1} \partial y_{2}}\right|_{(0,0,0)}=-\delta_{2}, \\
a_{11}= & \left.\frac{\partial^{2} F_{1}(\widetilde{\psi}(\bar{Y}))}{\partial^{2} y_{1}^{2}}\right|_{(0,0,0)} \\
= & \frac{\left(-c^{2} k m^{3} s^{3}+2 c k m^{2} p s^{3}-3 c^{2} k m^{2} s^{2}-k m p^{2} s^{3}\right) \delta_{2}}{(c m s-p s+c)^{2}(m s+1)} \\
& +\frac{\left(c^{2} E_{0} m+2 c k p s-c^{2} k-c E_{0} p\right) \delta_{2}}{(c m s-p s+c)^{2}(m s+1)} \\
& +\frac{\left(4 c k m p s^{2}-3 c^{2} k m s-k p^{2} s^{2}\right) \delta_{2}}{(c m s-p s+c)^{2}(m s+1)}, \\
a_{13}= & \left.\frac{\partial^{2} F_{1}(\widetilde{\psi}(\bar{Y}))}{\partial y_{1} \partial \delta^{*}}\right|_{(0,0,0)}=s\left(-k+\frac{E_{0} m}{(1+m s)^{2}}\right)+\frac{s E_{0} p}{(1+m s)^{2}((p-c m) s-c)}, \\
a_{23}= & \left.\frac{\partial^{2} F_{1}(\widetilde{\psi}(\bar{Y}))}{\partial y_{2} \partial \delta^{*}}\right|_{(0,0,0)}=-s, \\
a_{111}= & \left.\frac{\partial^{3} F_{1}(\widetilde{\psi}(\bar{Y}))}{\partial^{3} y_{1}}\right|_{(0,0,0)}=-\frac{\left(c^{2} m^{2}-2 c m p+p^{2}\right) E_{0} c \delta_{2}}{(c m s-p s+c)^{3}(1+m s)}, \\
a_{113}= & \left.\frac{\partial^{3} F_{1}(\widetilde{\psi}(\bar{Y}))}{\partial^{2} y_{1} \partial \delta^{*}}\right|_{(0,0,0)} \\
= & \frac{-c^{2} k m^{3} s^{3}+2 c k m^{2} p s^{3}-3 c^{2} k m^{2} s^{2}-k m p^{2} s^{3}}{(c m s-p s+c)^{2}(m s+1)} \\
& +\frac{4 c k m p s^{2}-3 c^{2} k m s-k p^{2} s^{2}+c^{2} E_{0} m}{(c m s-p s+c)^{2}(m s+1)} \\
& +\frac{2 c k p s-c^{2} k-c E_{0} p}{(c m s-p s+c)^{2}(m s+1)}, \quad b_{123}=\left.\frac{\partial^{3} F_{1}(\widetilde{\psi}(\bar{Y}))}{\partial y_{1} \partial y_{2} \delta^{*}}\right|_{(0,0,0)}=1 . \\
a_{123}= & \left.\frac{\partial^{3} F_{1}(\widetilde{\psi}(\bar{Y}))}{\partial y_{1} \partial y_{2} \delta^{*}}\right|_{(0,0,0)}=-1, \quad b_{1}=\left.\frac{\partial F_{2}(\widetilde{\psi}(\bar{Y}))}{\partial y_{1}}\right|_{(0,0,0)}=\delta_{2} y_{0}, \\
b_{2}= & \left.\frac{\partial F_{2}(\widetilde{\psi}(\bar{Y}))}{\partial y_{2}}\right|_{(0,0,0)}=1, \quad b_{12}=\left.\frac{\partial^{2} F_{2}(\widetilde{\psi}(\bar{Y}))}{\partial y_{1} \partial y_{2}}\right|_{(0,0,0)}=\delta_{2}, \\
b_{13}= & \left.\left.\frac{\partial^{2} F_{2}(\widetilde{\psi}(\bar{Y}))}{\partial y_{1} \partial \delta^{*}}\right|_{(0,0,0)}=y_{0}, \quad b_{1}\right)
\end{aligned}\right.
$$

Construct an invertible matrix

$$
\begin{aligned}
& T=\left(\begin{array}{ccc}
t_{11} & t_{12} & 0 \\
1 & 1 & 0 \\
0 & 0 & 1
\end{array}\right), \\
& T^{-1}=\left(\begin{array}{ccc}
\tilde{t}_{11} & \tilde{t}_{12} & 0 \\
\tilde{t}_{21} & \tilde{t}_{22} & 0 \\
0 & 0 & 1
\end{array}\right),
\end{aligned}
$$

where

$$
\begin{aligned}
& t_{11}=\frac{a_{2}}{\frac{1}{2}-\frac{1}{2} a_{1}+\frac{1}{2} \sqrt{a_{1}^{2}+4 a_{2} b_{1}-2 a_{1}+1}}, \\
& t_{12}=\frac{a_{2}}{\frac{1}{2}-\frac{1}{2} a_{1}-\frac{1}{2} \sqrt{a_{1}^{2}+4 a_{2} b_{1}-2 a_{1}+1}}, \\
& \tilde{t}_{11}=\frac{1}{4} \frac{\left(1-a_{1}+\sqrt{a_{1}^{2}+4 a_{2} b_{1}-2 a_{1}+1}\right)\left(-1+a_{1}+\sqrt{a_{1}^{2}+4 a_{2} b_{1}-2 a_{1}+1}\right)}{a_{2} \sqrt{a_{1}^{2}+4 a_{2} b_{1}-2 a_{1}+1}}, \\
& \tilde{t}_{12}=\frac{1}{2} \frac{1-a_{1}+\sqrt{a_{1}^{2}+4 a_{2} b_{1}-2 a_{1}+1}}{\sqrt{a_{1}^{2}+4 a_{2} b_{1}-2 a_{1}+1}}, \\
& \tilde{t}_{21}=-\frac{1}{4} \frac{\left(1-a_{1}+\sqrt{a_{1}^{2}+4 a_{2} b_{1}-2 a_{1}+1}\right)\left(-1+a_{1}+\sqrt{a_{1}^{2}+4 a_{2} b_{1}-2 a_{1}+1}\right)}{a_{2} \sqrt{a_{1}^{2}+4 a_{2} b_{1}-2 a_{1}+1}},
\end{aligned}
$$




$$
\tilde{t}_{22}=\frac{1}{2} \frac{-1+a_{1}+\sqrt{a_{1}^{2}+4 a_{2} b_{1}-2 a_{1}+1}}{\sqrt{a_{1}^{2}+4 a_{2} b_{1}-2 a_{1}+1}} .
$$

Now, make the translation

$$
\left(\begin{array}{l}
y_{1} \\
y_{2} \\
\delta^{*}
\end{array}\right)=T\left(\begin{array}{l}
z_{1} \\
z_{2} \\
\delta^{*}
\end{array}\right)
$$

to system (4.6), then we get

$$
\left(\begin{array}{c}
z_{1} \\
z_{2} \\
\delta^{*}
\end{array}\right) \rightarrow\left(\begin{array}{ccc}
-1 & 0 & 0 \\
0 & \lambda_{2} & 0 \\
0 & 0 & 1
\end{array}\right)\left(\begin{array}{c}
z_{1} \\
z_{2} \\
\delta^{*}
\end{array}\right)+\left(\begin{array}{c}
\varphi_{1}\left(z_{1}, z_{2}, \delta^{*}\right) \\
\varphi_{2}\left(z_{1}, z_{2}, \delta^{*}\right) \\
0
\end{array}\right)
$$

where

$$
\left\{\begin{aligned}
\varphi_{1}\left(z_{1}, z_{2}, \delta^{*}\right)= & \alpha_{11} z_{1}^{2}+\alpha_{22} z_{2}^{2}+\alpha_{12} z_{1} z_{2}+\alpha_{13} z_{1} \delta^{*}+\alpha_{23} z_{2} \delta^{*}+\alpha_{123} z_{1} z_{2} \delta^{*}+\alpha_{113} z_{1}^{2} \delta^{*} \\
& +\alpha_{223} z_{2}^{2} \delta^{*}+\alpha_{111} z_{1}^{3}+\alpha_{222} z_{2}^{3}+\alpha_{112} z_{1}^{2} z_{2}+\alpha_{122} z_{1} z_{2}^{2}+\alpha_{133} z_{1} \delta^{* 2} \\
& +\alpha_{233} z_{2} \delta^{* 2}+O\left(\left(\left|z_{1}\right|+\left|z_{2}\right|+\left|\delta^{*}\right|\right)^{4}\right), \\
\varphi_{2}\left(z_{1}, z_{2}, \delta^{*}\right)= & \beta_{11} z_{1}^{2}+\beta_{22} z_{2}^{2}+\beta_{12} z_{1} z_{2}+\beta_{13} z_{1} \delta^{*}+\beta_{23} z_{2} \delta^{*}+\beta_{123} z_{1} z_{2} \delta^{*}+\beta_{113} z_{1}^{2} \delta^{*} \\
& +\beta_{223} z_{2}^{2} \delta^{*}+\beta_{133} z_{1} \delta^{* 2}+\beta_{233} z_{2} \delta^{* 2}+O\left(\left(\left|z_{1}\right|+\left|z_{2}\right|+\left|\delta^{*}\right|\right)^{4}\right),
\end{aligned}\right.
$$

with

$$
\left\{\begin{array}{l}
\alpha_{11}=\tilde{t}_{11} a_{11} t_{11}^{2}+\tilde{t}_{11} a_{12} t_{11}+\tilde{t}_{12} b_{12} t_{11}, \\
\alpha_{12}=2 \tilde{t}_{11} a_{11} t_{11} t_{12}+\tilde{t}_{11} a_{12} t_{11}+\tilde{t}_{11} a_{12} t_{12}+\tilde{t}_{12} b_{12} t_{11}+\tilde{t}_{12} b_{12} t_{12}, \\
\alpha_{13}=\tilde{t}_{11} a_{13} t_{11}+\tilde{t}_{12} b_{13} t_{11}+\tilde{t}_{11} a_{23}, \quad \alpha_{22}=\tilde{t}_{11} a_{11} t_{12}^{2}+\tilde{t}_{11} a_{12} t_{12}+\tilde{t}_{12} b_{12} t_{12} \\
\alpha_{23}=\tilde{t}_{11} a_{13} t_{12}+\tilde{t}_{12} b_{13} t_{12}+\tilde{t}_{11} a_{23}, \quad \alpha_{111}=\tilde{t}_{11} a_{111} t_{11}^{3}, \quad \alpha_{112}=3 \tilde{t}_{11} a_{111} t_{11}^{2} t_{12} \\
\alpha_{113}=\tilde{t}_{11} a_{113} t_{11}^{2}+\tilde{t}_{11} a_{123} t_{11}+\tilde{t}_{12} b_{123} t_{11}+\tilde{t}_{11} a_{223}, \quad \alpha_{122}=3 \tilde{t}_{11} a_{111} t_{11} t_{12}^{2} \\
\alpha_{123}=2 \tilde{t}_{11} a_{113} t_{11} t_{12}+\tilde{t}_{11} a_{123} t_{11}+\tilde{t}_{11} a_{123} t_{12}+\tilde{t}_{12} b_{123} t_{11}+\tilde{t}_{12} b_{123} t_{12}+2 \tilde{t}_{11} b_{223} \\
\alpha_{133}=\tilde{t}_{11} a_{233}, \quad \alpha_{222}=\tilde{t}_{11} a_{111} t_{12}^{3}, \\
\alpha_{223}=\tilde{t}_{11} a_{113} t_{12}^{2}+\tilde{t}_{11} a_{123} t_{12}+\tilde{t}_{12} b_{123} t_{12}+\tilde{t}_{11} a_{223}, \\
\alpha_{233}=\tilde{t}_{11} a_{233},
\end{array}\right.
$$

and

$$
\left\{\begin{array}{l}
\beta_{11}=\tilde{t}_{21} a_{11} t_{11}^{2}+\tilde{t}_{21} a_{12} t_{11}+\tilde{t}_{22} b_{12} t_{11}, \\
\beta_{12}=2 \tilde{t}_{21} a_{11} t_{11} t_{12}+\tilde{t}_{21} a_{12} t_{11}+\tilde{t}_{21} a_{12} t_{12}+\tilde{t}_{22} b_{12} t_{11}+\tilde{t}_{22} b_{12} t_{12}, \\
\beta_{13}=\tilde{t}_{21} a_{13} t_{11}+\tilde{t}_{22} b_{13} t_{11}+\tilde{t}_{21} a_{23}, \quad \beta_{22}=\tilde{t}_{21} a_{11} t_{12}^{2}+\tilde{t}_{21} a_{12} t_{12}+\tilde{t}_{22} b_{12} t_{12}, \\
\beta_{23}=\tilde{t}_{21} a_{13} t_{12}+\tilde{t}_{22} b_{13} t_{12}+\tilde{t}_{21} a_{23}, \\
\beta_{113}=\tilde{t}_{21} a_{113} t_{11}^{2}+\tilde{t}_{21} a_{123} t_{11}+\tilde{t}_{22} b_{123} t_{11}+\tilde{t}_{21} a_{223}, \\
\beta_{123}=2 \tilde{t}_{21} a_{113} t_{11} t_{12}+2 \tilde{t}_{21} a_{223}+\tilde{t}_{21} a_{123} t_{11}+\tilde{t}_{21} a_{123} t_{12}+\tilde{t}_{22} b_{123} t_{11}+\tilde{t}_{22} b_{123} t_{12}, \\
\beta_{133}=\tilde{t}_{21} a_{233}, \quad \beta_{223}=\tilde{t}_{21} a_{113} t_{12}^{2}+\tilde{t}_{21} a_{123} t_{12}+\tilde{t}_{22} b_{123} t_{12}+\tilde{t}_{21} a_{223}, \\
\beta_{233}=\tilde{t}_{21} a_{233} .
\end{array}\right.
$$


Having gone through these coordinate changes, the parameter-dependent center manifold of (4.7) can be approximately represented as follows:

$$
\begin{aligned}
& W^{c}(0,0,0)=\left\{\left(z_{1}, z_{2}, \delta^{*}\right) \in R^{3} \mid z_{2}=h\left(z_{1}, \delta^{*}\right), h(0,0)=0, D h(0,0)=0\right\}, \\
& h\left(z_{1}, \delta^{*}\right)=c_{0} \delta^{* 2}+c_{1} z_{1} \delta^{*}+c_{2} z_{1}^{2}+O\left(\left(\left|z_{1}\right|+\left|\delta^{*}\right|\right)^{3}\right) .
\end{aligned}
$$

Since the center manifold is invariant under the flow, one can substitute $z_{2}=h\left(z_{1}, \delta^{*}\right)$ into the second formula of (4.7) and obtain

$$
h\left(-z_{1}+\varphi_{1}\left(z_{1}, h\left(z_{1}, \delta^{*}\right), \delta^{*}\right), \delta^{*}\right)-\lambda_{2} h\left(z_{1}, \delta^{*}\right)-\varphi_{2}\left(z_{1}, h\left(z_{1}, \delta^{*}\right), \delta^{*}\right)=0 .
$$

Substituting (4.8) into (4.9), and then comparing the coefficients for $\delta^{* 2}, \delta^{*} z_{1}$ and $z_{1}^{3}$, we get

$$
c_{0}=0, \quad c_{1}=-\frac{\beta_{13}}{1+\lambda_{2}}, \quad c_{2}=\frac{\beta_{111}}{1-2 \alpha_{11}-\beta_{12}}=0 .
$$

Therefore, we consider the map which is (4.7) restricted to the center manifold $W^{c}(0,0)$,

$$
\begin{aligned}
F: z_{1} \rightarrow & -z_{1}+d_{11} z_{1}^{2}+d_{12} z_{1} \delta^{*}+d_{112} z_{1}^{2} \delta^{*}+d_{122} z_{1} \delta^{* 2}+d_{22} \delta^{* 2}+d_{111} z_{1}^{3}+d_{222} \delta^{* 3} \\
& +O\left(\left(\left|z_{1}\right|+\left|\delta^{*}\right|\right)^{4}\right)
\end{aligned}
$$

where

$$
\begin{aligned}
& d_{11}=\alpha_{11}, \quad d_{112}=\alpha_{113}+\alpha_{12} c_{1}, \quad d_{22}=\alpha_{33}, \quad d_{222}=\alpha_{333}, \quad d_{12}=\alpha_{13}, \\
& d_{122}=\alpha_{133}+\alpha_{23} c_{1}, \quad d_{111}=\alpha_{111} .
\end{aligned}
$$

In order for system (4.10) to undergo a flip bifurcation at the origin, we require that the two quantities

$$
\tilde{\alpha}_{1}=\left(2 \frac{\partial F}{\partial \delta^{*} \partial z_{1}}+\frac{\partial F}{\partial \delta^{*}} \frac{\partial F}{\partial z_{1}}\right)_{(0,0)}=2 d_{12}
$$

and

$$
\tilde{\alpha}_{2}=\left(\frac{1}{2}\left(\frac{\partial^{2} F}{\partial z_{1}^{2}}\right)^{2}+\frac{1}{3}\left(\frac{\partial^{3} F}{\partial z_{1}^{3}}\right)\right)_{(0,0)}=2 d_{11}^{2}+2 d_{111}
$$

do not equal zero [22].

Let

$$
\Omega_{2}=\left\{(a, k, s, p, m, c, v, \delta) \mid(a, k, s, p, m, c, v) \in \pi, 0<\delta=\delta_{2}, \delta \Lambda_{2}-\delta \Lambda_{1} \neq 2,4\right\},
$$

then we have the following result.

Theorem 4.2 Assuming that $(a, k, s, p, m, c, v, \delta) \in \Omega_{2}$. If $\tilde{\alpha}_{2} \neq 0$, then the system (2.3) undergoes a flip bifurcation at the fixed point $\left(x_{0}, y_{0}, E_{0}\right)$ when the bifurcation parameter $\delta$ varies in a small neighborhood of $\delta_{2}$. Moreover, if $\tilde{\alpha}_{2}>0$ (resp., $\left.\tilde{\alpha}_{2}<0\right)$, then the period-2 orbits that bifurcate from $\left(x_{0}, y_{0}, E_{0}\right)$ are stable (resp., unstable). 


\section{Numerical simulations}

In this section, we give two examples to illustrate our theoretical analysis.

Example 5.1 (Neimark-Sacker bifurcation) Here we present a numerical analysis of the proposed system (2.3) with the following artificially chosen data: $a=4, k=1, s=2, p=1$, $m=0.1, c=1$ and $v=0.6$ with $(a, k, s, p, m, c, v) \in \pi$. It is easy to verify that the system (2.3) has a unique fixed point $(2,1.25,0.9)$ and $\delta_{1}=0.125$.

The multipliers of the positive fixed point are $\lambda_{ \pm} \approx 0.9805 \pm 0.1967 i$ with $\left|\lambda_{ \pm}\right|=1$ and $\alpha=0.1563>0$. Since $H=0.0391 \neq 2,3,4$, the condition (4.1) holds. Then, using Theorem 4.1, the system (2.3) undergoes a Neimark-Sacker bifurcation at the fixed point $(2,1.25,0.9)$ with $l=0.0017>0$.

Figures 1,2 and 3 reveal that the fixed point $(2,1.25,0.9)$ of $(2.3)$ is unstable for $\delta=$ $0.125+0.0014$ and becomes stable for $\delta=0.125-0.035$, and a repelling limit cycle appears around it at $\delta=0.125-0.0001$.

Example 5.2 (Flip bifurcation) Fix $a=16, k=4, s=3.9, p=7, m=1, c=1$ and $v=1$ and the fixed point $(3.9,0.3554,0.2188)$ with $(a, k, s, p, m, c, v) \in \pi$. After computation we get the following quantities:

$$
\begin{aligned}
& \delta_{2}=0.1294, \\
& \tilde{\alpha}_{1}=-0.1792, \\
& \tilde{\alpha}_{2}=0.0022-2 \times 6.8573 \times 10^{-6}>0 .
\end{aligned}
$$
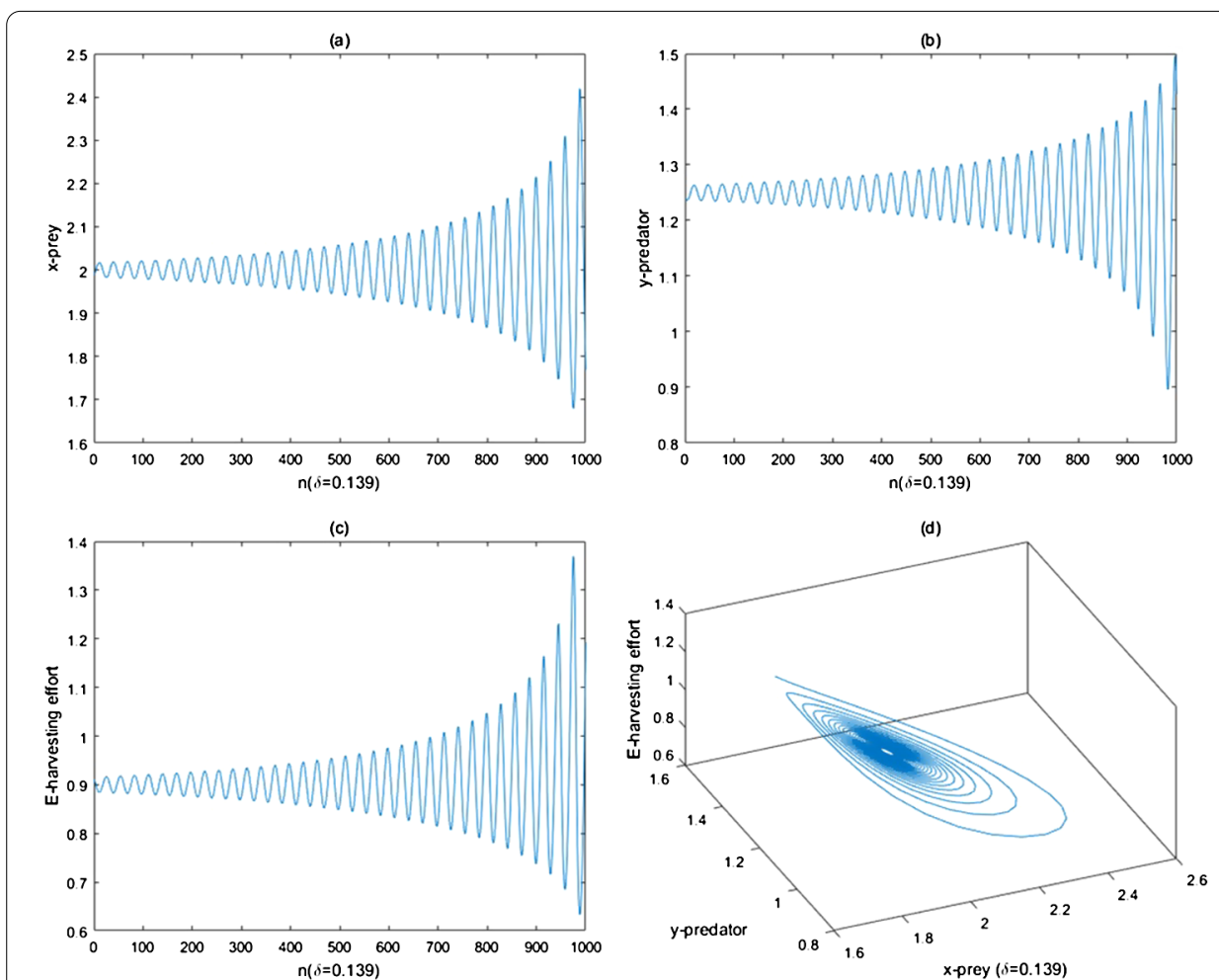

Figure $1 \delta=\delta_{1}+0.014$, the fixed point $(2,1.25,0.9)$ is an unstable focus 

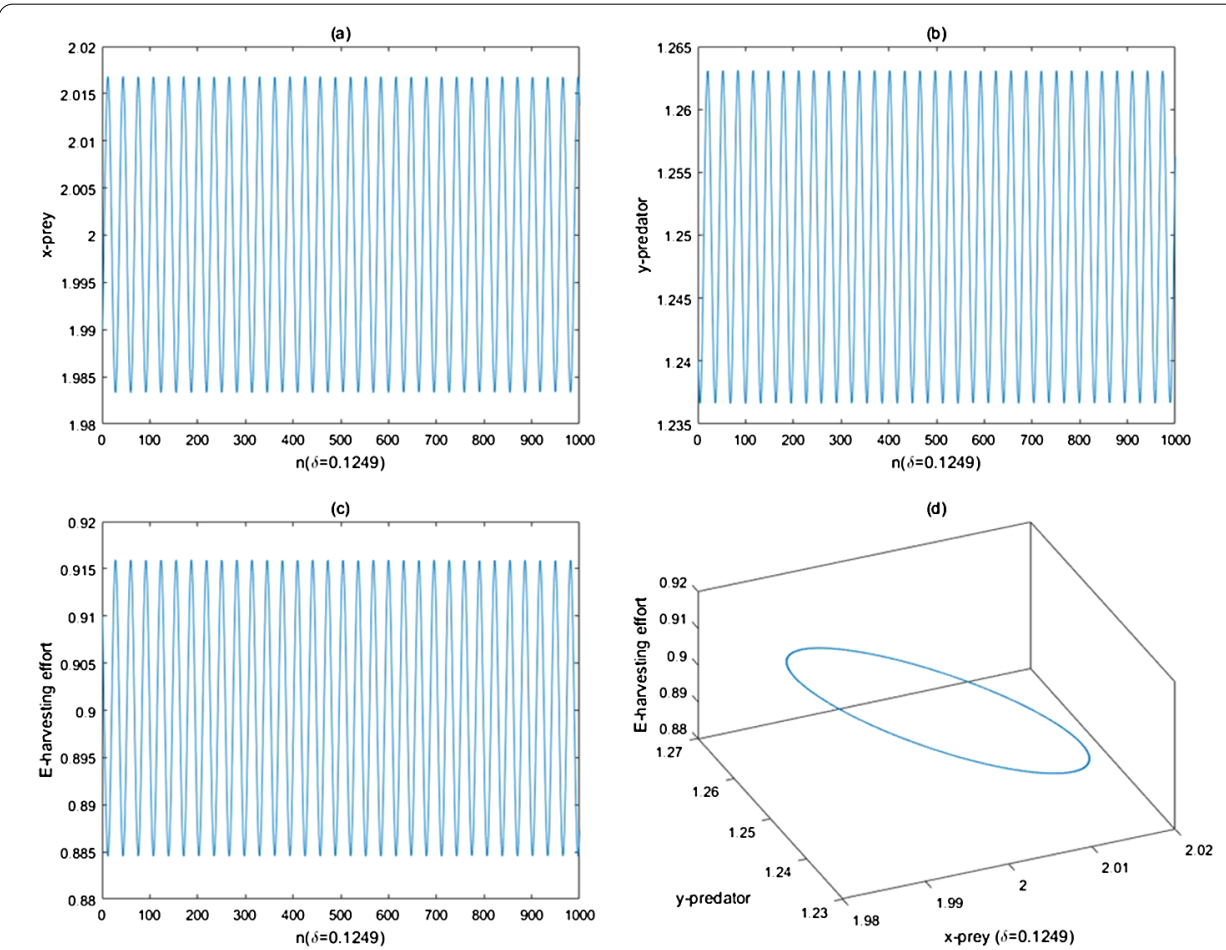

Figure $2 \delta=\delta_{1}-0.0001$. An repelling invariant closed curve bifurcates from the fixed point $(2,1.25,0.9)$
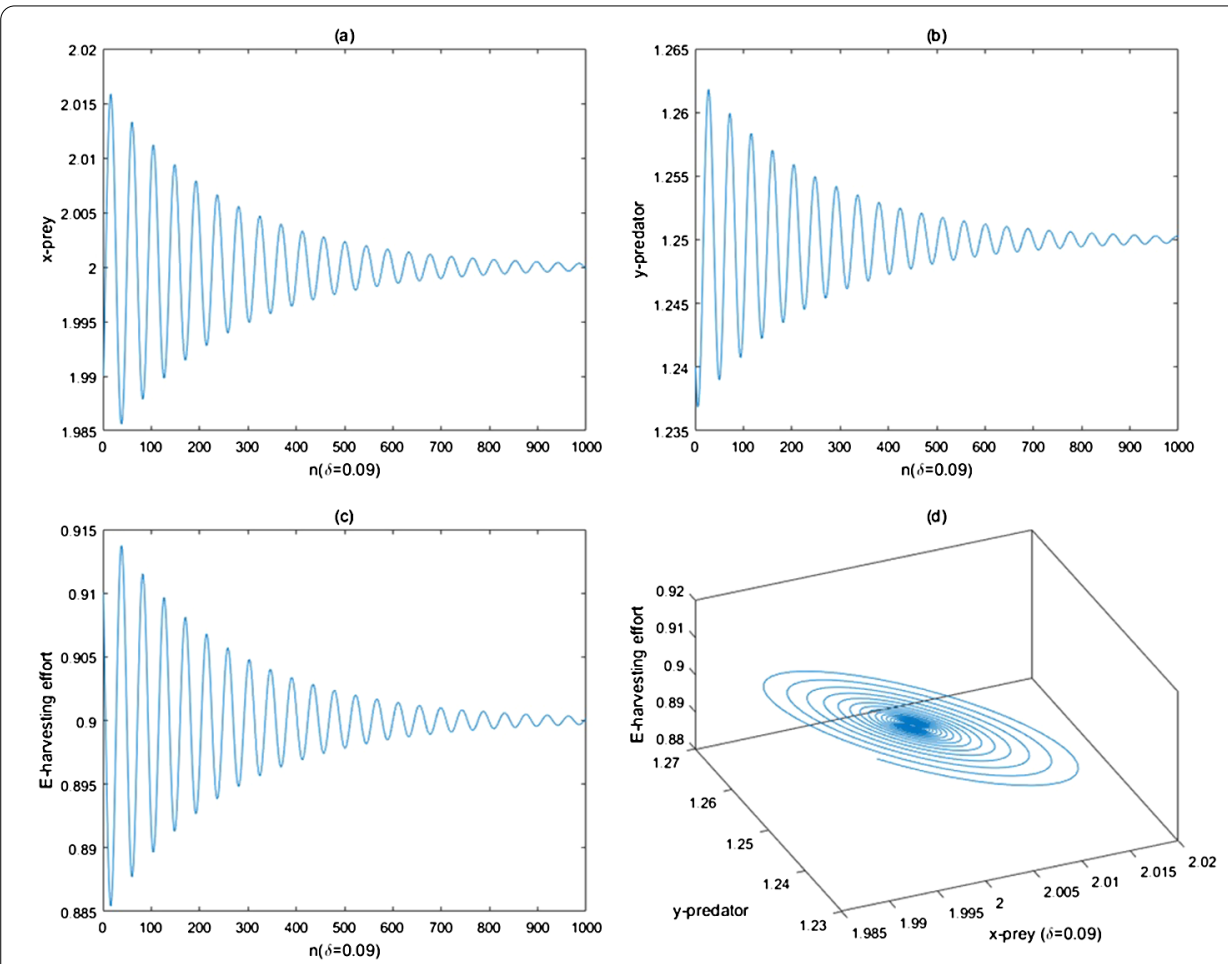

Figure $3 \delta=\delta_{1}-0.035$, the fixed point $(2,1.25,0.9)$ is a stable focus 


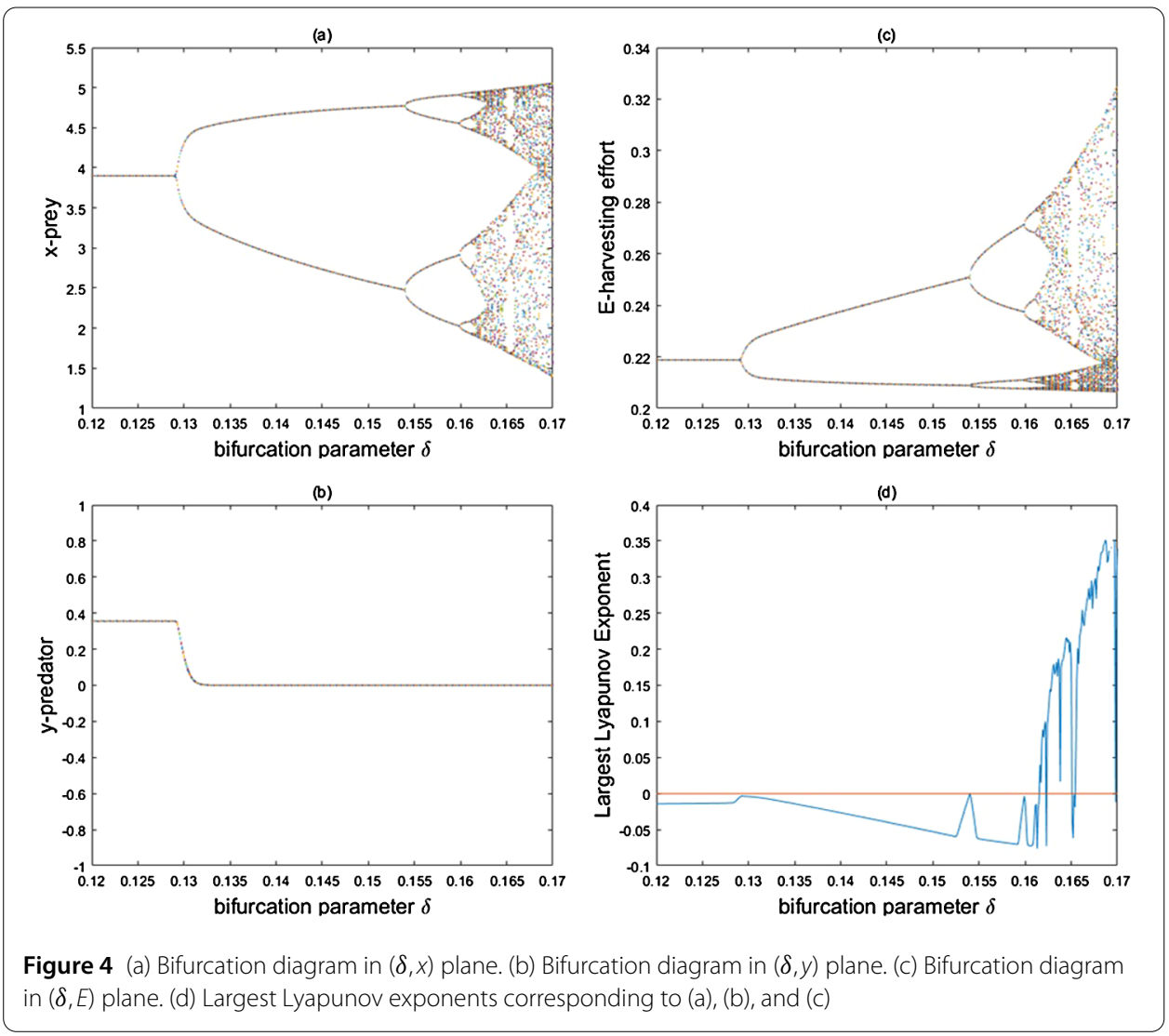

Figures 4(a), 4(b) and 4(c) reveal that the fixed point is stable for $\delta<0.1294$, and loses its stability at the flip bifurcation parameter value $\delta=0.1294$. We also observe that there is a cascade of period doubling bifurcations. The largest Lyapunov exponents corresponding to Figs. 4(a), 4(b) and 4(c) are computed in Fig. 4(d).

Figure 5 shows the phase portraits which are associated with Figs. 4(a), 4(b) and 4(c). For $\delta \in(0.12,0.1616)$, there are orbits of period $2,4,8, \ldots$ When $\delta=0.1616$, one can see that the largest Lyapunov exponent is positive, thereby confirming that the system is chaotic. And then for $\delta \in(0.1616,017)$ some largest Lyapunov exponents are negative, indicating the existence of periodic windows in the chaos region.

\section{Conclusion}

The present paper is concerned with the dynamics of a discrete-time economic predatorprey system in the presence of a type of nonlinear harvesting function. We find the fixed point and its stability. Most interestingly, we have seen that our results reveal a far richer dynamics of the discrete model compared with the continuous one proposed in [9], including an invariant circle, cascades of period-doubling bifurcation and chaotic sets. We confirm the complexity of the dynamic behavior by computing the largest Lyapunov exponents. This paper extends our previous works (see $[8,9])$ and provides a sufficiently general parameterization method for a wide range of discrete singular systems.

However, in the presented harvesting function, we have no concern about the effects of competition between boats which will increase the complexity of the normal form and the amount of calculation. Moreover, for discrete-time systems, Marotto's theorem [23, 24] 


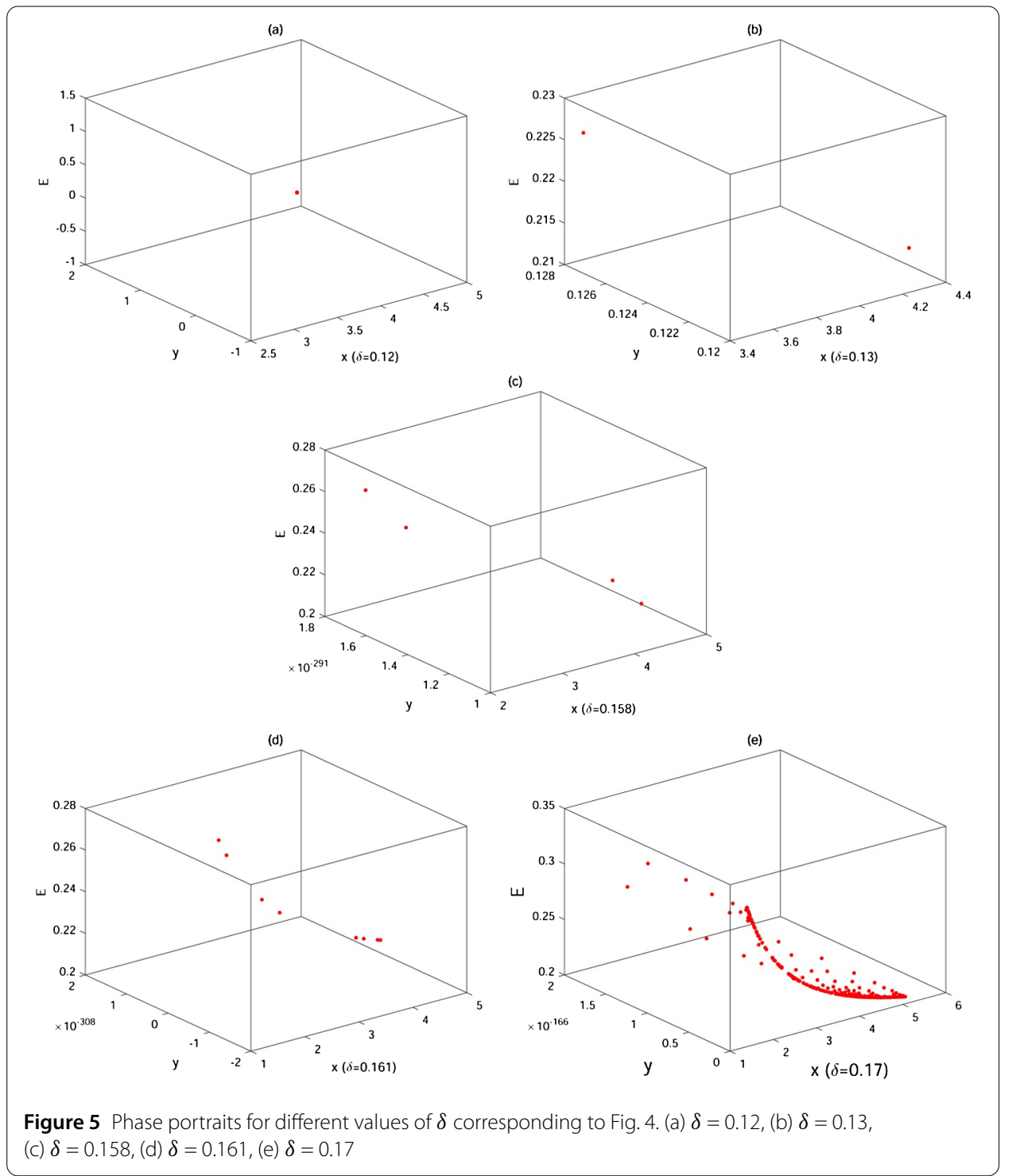

is a sufficient criterion for the existence of chaos. If the fixed point of system (2.3) is a snap-back repeller under certain parameter conditions, then one can conclude that the system is chaotic in the Marotto sense. Furthermore, various feedback controls can be implemented for controlling the bifurcation and chaos in the system (2.3), which should be useful for fishery management control and biological conversion. Space prevents that discussion here, but these issues will be the topics of our future research.

Acknowledgements

The authors wish to express their gratitude to the editors and reviewers for the helpful comments. This research is supported by National Natural Science Foundation of China under grant No. 71271158.

Competing interests

The authors declare that they have no competing interests.

Authors' contributions

The study presented here was carried out in collaboration between all authors. All authors read and approved the final manuscript. 


\section{Publisher's Note}

Springer Nature remains neutral with regard to jurisdictional claims in published maps and institutional affiliations.

Received: 25 January 2018 Accepted: 5 April 2018 Published online: 12 April 2018

\section{References}

1. Clark, C.W.: Mathematical Bioeconomics: The Optimal Management of Renewable Resources. Wiley, New York (2010)

2. Chen, B.S., Chen, J.J.: Complex dynamic behaviors of a discrete predator-prey model with stage structure and harvesting. Int. J. Biomath. 10(1), 1-25 (2017)

3. Gupta, R.P., Chandra, P.: Dynamical properties of a prey-predator-scavenger model with quadratic harvesting. Commun. Nonlinear Sci. Numer. Simul. 49, 202-214 (2017)

4. Li, M., Chen, B.S., Ye, H.W.: A bioeconomic differential algebraic predator-prey model with nonlinear prey harvesting. Appl. Math. Model. 42, 17-28 (2017)

5. Liu, C., Lu, N., Zhang, Q.L.: Dynamical analysis in a hybrid bioeconomic system with multiple time delays and strong Allee effect. Math. Comput. Simul. 136, 104-131 (2017)

6. Liu, W., Fu, C.J., Chen, B.S.: Hopf bifurcation and center stability for a predator-prey biological economic model with prey harvesting. Commun. Nonlinear Sci. Numer. Simul. 17(10), 3989-3998 (2012)

7. Liu, W., Jiang, Y.L.: Bifurcation of a delayed Gauss predator-prey model with Michaelis-Menten type harvesting. J. Theor. Biol. 438, 116-132 (2018)

8. Liu, W.Y., Fu, C.J., Chen, B.S.: Stability and Hopf bifurcation of a predator-prey biological economic system with nonlinear harvesting rate. Int. J. Nonlinear Sci. Numer. Simul. 16(6), 249-258 (2015)

9. Liu, W.Y., Li, B.W., Fu, C.J., Chen, B.S.: Dynamics of a predator-prey ecological system with nonlinear harvesting rate Wuhan Univ. J. Nat. Sci. 20(1), 25-33 (2015)

10. Kolmogorov, A.N.: Sulla teoria di Volterra della Lotta per l'esistenza. G. Ist. Ital. Attuari 7, 74-80 (1936) (in Italian)

11. Santra, P., Mahapatra, G.S., Pal, D.: Analysis of differential-algebraic prey-predator dynamical model with super predator harvesting on economic perspective. Int. J. Dyn. Control 4, 266-274 (2016)

12. Zhang, X., Zhang, Q.L.: Bifurcation analysis and control of a class of hybrid biological economic models. Nonlinear Anal. Hybrid Syst. 3, 578-587 (2009)

13. Seijo, J.C., Defeo, O., Salas, S.: Fisheries Bioeconomics: Theory, Modelling and Management. Fao Fisheries Technical Papers, vol. 368. Food and Agriculture Organization of the United Nation, New York (1998)

14. Shelton, S.J., Myers, R.A., Dunn, A.: Is catch-per-unit effort proportional to abundance? Can. J. Fish. Aquat. Sci. 58 1760-1772 (2001)

15. Kot, M.: Elements of Mathematical Ecology. Cambridge University Press, Cambridge (2001)

16. Potra, F.A., Yen, J.: Implicit numerical integration for Euler-Lagrange equations via tangent space parametrization. Circuits Syst. Signal Process. 19(1), 77-98 (1991)

17. Agnew, T.T.: Optimal exploitation of a fishery employing a nonlinear harvesting function. Ecol. Model. 6, 47-57 (1979)

18. Fred, B., Carlos, C.C.: Mathematical Models in Population Biology and Epidemiology. Springer, New York (2011)

19. Goh, B.S.: Management and Analysis of Biological Populations. Developments in Agricultural and Managed-Forest Ecology. Elsevier, Amsterdam (1980)

20. Wiggins, S.: Introduction to Applied Nonlinear Dynamical Systems and Chaos. Springer, New York (2003)

21. Liu, X.L., Xiao, D.M.: Complex dynamic behaviors of a discrete-time predator-prey system. Chaos Solitons Fractals 32, 80-94 (2007)

22. Guckenheimer, J., Holmes, P.: Nonlinear Oscillations, Dynamical Systems, and Bifurcations of Vector Fields. Springer, New York (2002)

23. Marotto, F.R.: On redefining a snap-back repeller. Chaos Solitons Fractals 25(1), $25-28$ (2005)

24. Marotto, F.R.: Snap-back repellers imply chaos in $R^{n}$. J. Math. Anal. Appl. 63(1), 199-223 (1978)

\section{Submit your manuscript to a SpringerOpen ${ }^{\circ}$ journal and benefit from:}

- Convenient online submission

- Rigorous peer review

- Open access: articles freely available online

- High visibility within the field

- Retaining the copyright to your article

Submit your next manuscript at $\boldsymbol{~ s p r i n g e r o p e n . c o m ~}$ 